\title{
Behavioral and Biochemical Assessment of Monoaminergic Neural Transplants to Rat Frontal Neocortex
}

\author{
Caryl E. Sortwell and Jacqueline Sagen \\ Department of Anatomy and Cell Biology, University of Illinois at Chicago, \\ 808 S. Wood Street, Chicago, IL 60612, USA
}

The classical monoamine theory of depression states that depression may be caused by a central deficiency of biogenic amines, particularly norepinephrine and serotonin. It has been proposed that effective antidepressant therapies work by correcting the central deficiency in biogenic amines and reequilibrate monoaminergic systems in the direction of greater efficiency. The transplantation of pharmacologically relevant tissues to specific CNS sites is a novel approach toward restoring imbalanced functioning in the CNS. The aim of this study was to determine whether the transplantation of monoamine-producing tissues into the frontal neocortex of rats could alleviate depression in animal models.

In the first study, male Sprague-Dawley rats were transplanted with either catecholaminerich adrenal medulla, serotonin-rich pineal gland, a combination of both adrenal medulla and pineal gland, or an equal volume of striated muscle as a control implant that did not produce monoamines. The tissue was implanted stereotaxically into the frontal neocortex, a site shown previously to be a potent cortical site of action for antidepressant activity.

Six to eight weeks following surgery rats were assessed behaviorally using the Learned Helplessness (LH) model of depression. LH is widely considered to be one of the most reliable animal models of depression and is induced by exposure to inescapable and uncontrollable stress which results in deficits in subsequent performance when the stress is escapable and controllable. These deficits can be prevented by antidepressant therapies, such as imipramine. At different periods following termination of LH behavioral testing graft tissues were assessed morphologically using immunocytochemistry. Results of this first study revealed that $\mathrm{LH}$ was prevented in all three experimental monoamine implant groups but not prevented in the control muscle implanted rats. Immunocytochemical results show that the grafts survived well and continued to produce monoamines for periods up to six months.

In part two of the study, female SpragueDawley rats were assessed using the Forced Swimming Test (FST), a popular measure of antidepressant activity. In the FST rats are forced to swim in a confined plexiglass cylinder and after an initial energetic attempt to escape they assume a readily identifiable immobile posture. On subsequent immersion the rat assumes the immobile position more rapidly and the duration of immobility is recorded over a five minute period. This duration of immobility is significantly reduced by a wide variety of antidepressant treatments. Rats in this study were assessed using the FST immediately prior to surgery to determine baseline immobility scores. Next, rats were transplanted to the frontal cortex with either adrenal medullary tissue, pineal gland tissue or equal volumes of sciatic nerve. Sciatic nerve served as a control tissue that produces trophic factors but does not produce monoamines. A control non-implanted group was also used in this experiment. Six to eight weeks following transplantation rats were again assessed behaviorally with the FST and immobility scores were recorded. Following the termination of FST testing (eight weeks following graft implantation), blocks of cortical tissue, including the transplant region, were dissected out and extracted and analyzed for monoamine concentrations using high performance liquid chromatography (HPLC). 
Results from this study revealed that following transplantation, both pineal and adrenal medullary transplants significantly lowered immobility scores. In contrast to the monoamine transplant groups, the mean immobility scores for the sciatic nerve group and the nonimplanted group were not reduced from FST immobility scores prior to transplantation. Biochemical results showed that serotonin levels and norepinephrine levels, in the pineal implanted group and adrenal medullary implanted group, respectively, were significantly higher than both the sciatic implanted and the non-implanted groups.

In conclusion, these studies suggest that monoaminergic neural transplants can provide a local source of monoamines for antidepressive action. The ability of monoaminergic grafts to alleviate depression in two different animal models is a promising finding that adds credence to the possibility of monoaminergic neural transplants providing a new approach to the treatment of clinical depression. 

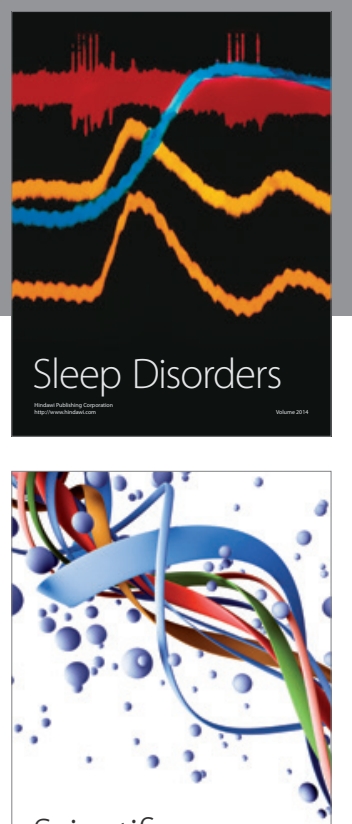

Scientifica
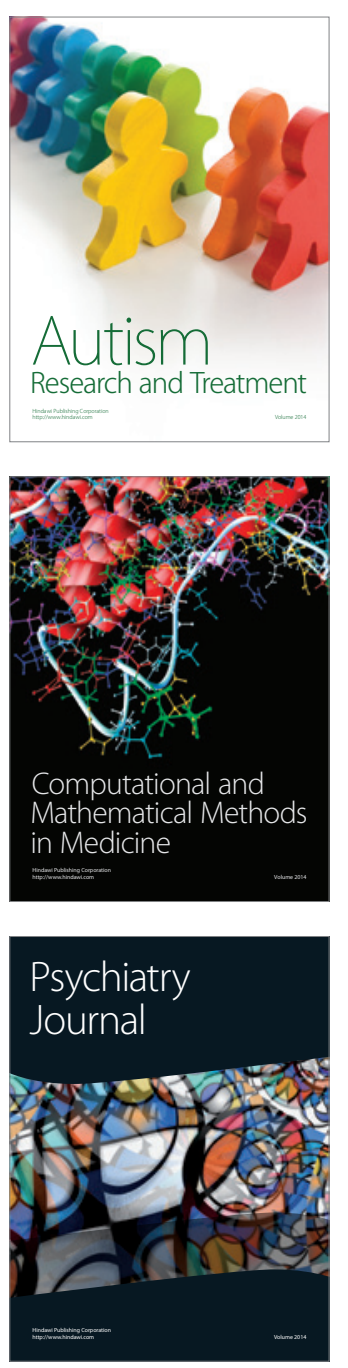
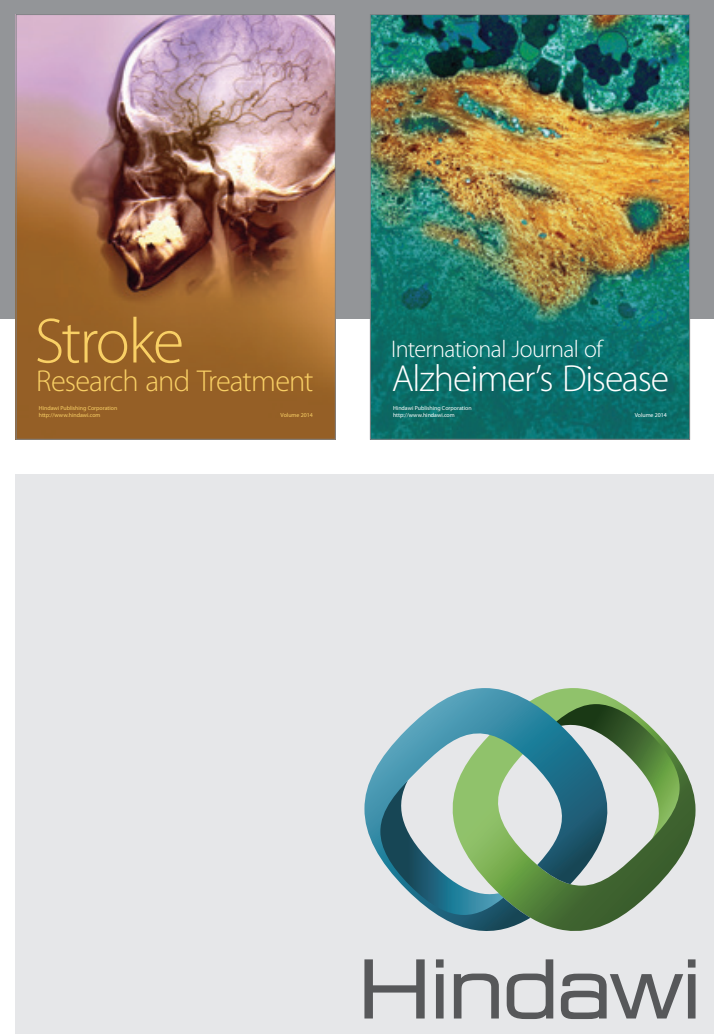

Submit your manuscripts at

http://www.hindawi.com
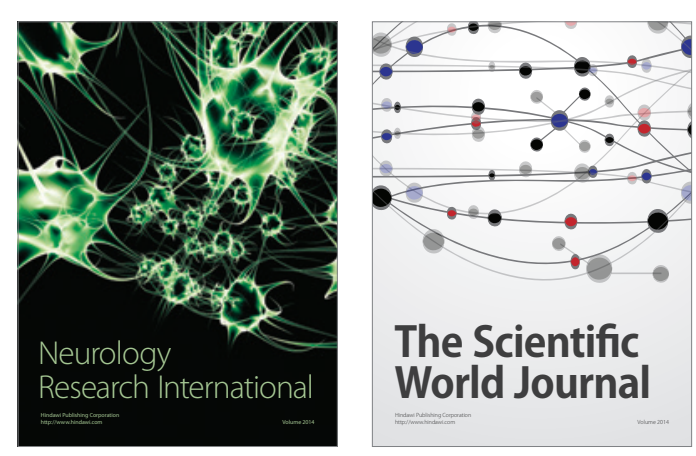

The Scientific World Journal

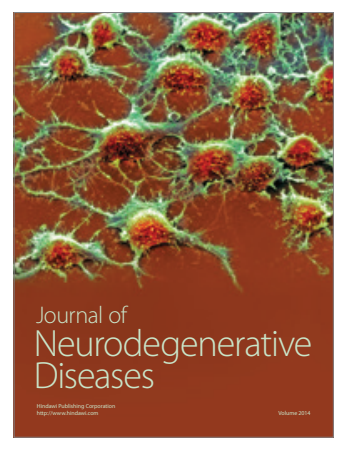

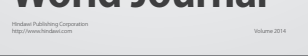

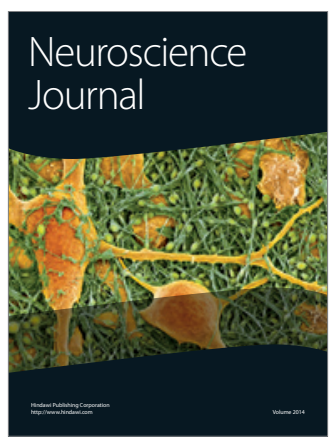

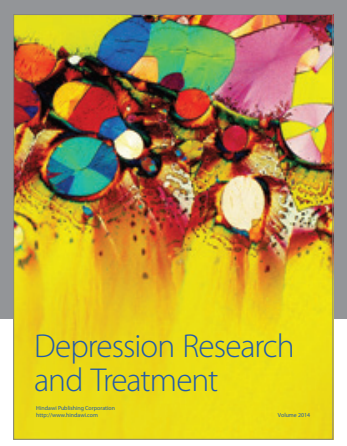
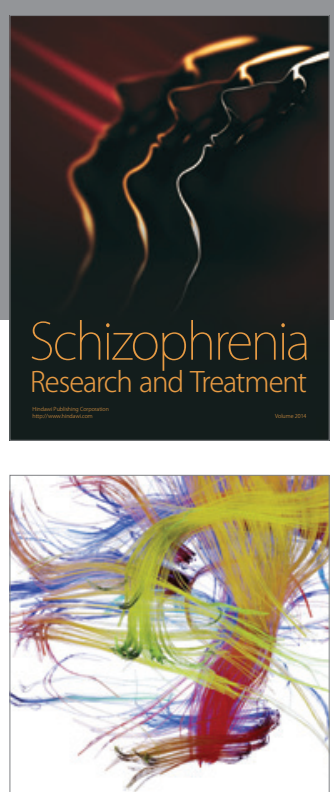

Brain Science

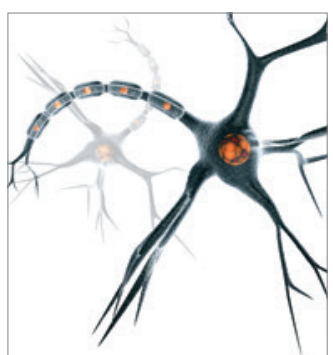

Neural Plasticity
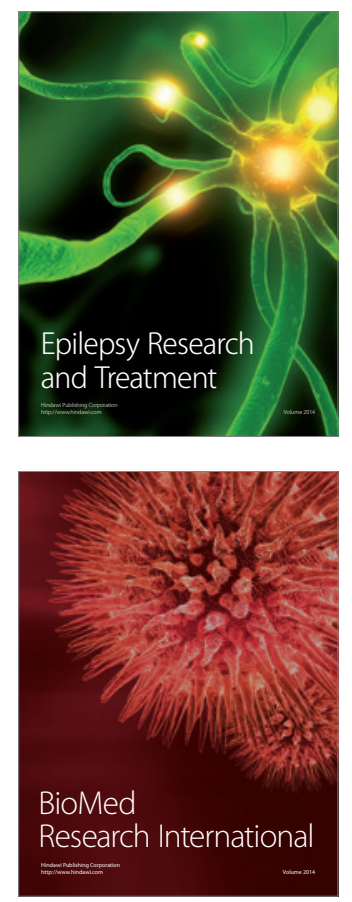

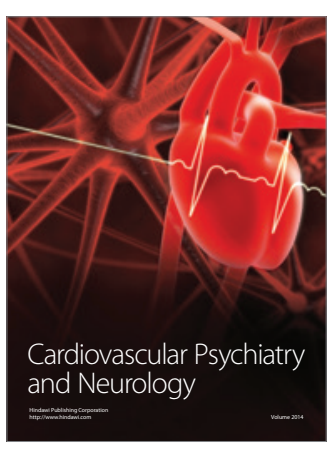

Parkinson's

Disease
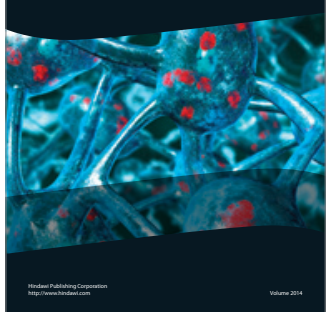